\title{
Functions of Wheel-rail Contact Geometry
}

\author{
Milan Bižicí ${ }^{*}$, Dragan Petrović ${ }^{1}$, Isidora Pančić ${ }^{2}$ \\ ${ }^{1}$ Faculty of Mechanical and Civil Engineering in Kraljevo, University of Kragujevac, Kraljevo (Serbia) \\ ${ }^{2}$ Faculty of Civil Engineering, University of Belgrade, Belgrade (Serbia)
}

The running of railway vehicles is based on the interaction of wheelset-track or wheel-rail with precisely defined nominal geometries. In this way, the geometric relationships which allowing smooth running in tangent and curved track are achieved. However, geometries in practice deviate from nominal to a greater or lesser extent. This cause the constant change of geometric relationships between wheels and rails and all dynamic parameters of railway vehicles during the running. For this reason, the modelling of wheel-rail contact geometry is very important task in rail vehicle dynamics. This paper gives a systematic approach to the analysis of wheelset-track and wheel-rail contact geometry. After introducing the parameters of wheelset and track geometry, the non-linear geometric relationships between wheel and rail profiles are analysed, and parameters of wheel-rail contact geometry are defined. In final stage, the functions which combines all analysed parameters are derived. A special discussion about the equivalent conicity as the most important function of wheel-rail contact geometry is given. The methods of its determination and influence on the dynamic behaviour of wheelset and vehicles are discussed. Considerations in the paper confirm the fact that wheel-rail contact geometry and equivalent conicty as function which representing it, play one of the most important role in dynamic behaviour of railway vehicles.

Keywords: functions, wheel-rail contact, geometry, equivalent conicity, railway vehicles

\section{INTRODUCTION}

The dynamic behaviour of railway vehicles is primarily related to the wheelset-track and wheel-rail interaction. In that sense, one of the most important task is modelling the geometrical relations between wheelset and track, and wheel and rail [1-3]. Many studies have shown that the wheel-rail contact geometry has a great influence on the quality of dynamic behaviour of railway vehicles. In the first instance, the contact geometry has significant influence on the wheel-rail surface stress distribution [4]. In this way, it affects the most important dynamic parameters of vehicle-track interaction - wheel-rail contact forces [5]. Accordingly, the contact geometry has significant influence on the ride comfort and running stability of railway vehicles [6, 7]. Many researches have shown that wheel-rail contact geometry play an important role in wheel-rail wear [8]. It is very important to note that many of these studies take into account only nominal contact geometry which is considered as constant. Given in mind that the contact geometry in practice more or less deviates from the nominal one, its importance in dynamic analysis of railway vehicles is even more pronounced [9]. Based on these considerations it can be concluded that the analysis and characterization of geometry of wheel-rail contact has very large importance in rail vehicle dynamics. Since the geometries of wheel and rail profiles are nonlinear, the problem is how to describe wheel-rail contact geometry during the running of railway vehicles [10]. In that sense, the task of this paper is systematic analysis of wheelset-track and wheel-rail contact geometry. This should result in obtaining the parameters of non-linear wheel-rail contact geometry, and in final stage, in functions which combines all analyzed parameters.

\section{PARAMETERS OF TRACK GEOMETRY}

It is very important to distinguish between nominal and real track geometry (track geometry in exploitation).
Nominal track geometry is defined by the international standards or standards of individual Railways which must be strictly observed during the design and construction of tracks. Each newly built track meets the prescribed nominal geometry within a certain allowable tolerance. However, after a certain period of exploitation due to the different influences (primarily wheel-rail contact forces) that starting geometry is degraded.

\subsection{Nominal track geometry}

The nominal track geometry is determined by the two groups of parameters - parameters of the cross-section and parameters of the line. The parameters of the crosssection are: track gauge, inclination, and cant.

The first and the most important parameter of the cross-section and the overall track geometry, is the track gauge $G$. It is measured $14 \mathrm{~mm}$ below the track plane, which is obtained by the connecting the upper points on the left and right rail (Fig. 1).

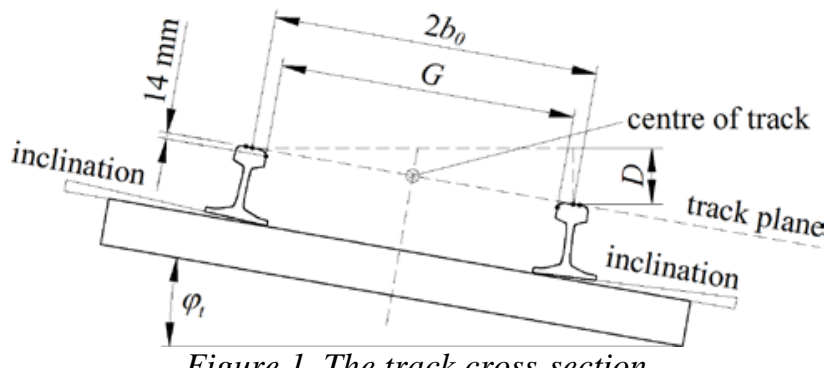

Figure 1. The track cross-section

Another very important parameter of the crosssection is rail inclination. As shown in Fig. 1, the rails are placed on the sleepers with a certain inclination towards the centre of the track. In combination with the wheel profile, this solution enables better transfer of load on the sleepers and ballast, as well as more uniform wear of wheel and rail contact surfaces. The third parameter of the cross-section is cant of outer rail in curve. If cant is 
marked with $D$, cant angle with $\varphi_{t}$, and distance between the nominal contact points with $2 b_{0}$ (Fig. 1 ), they satisfy the following relation:

$$
\sin \varphi_{t}=D / 2 b_{0}
$$

The parameters of the line are: radius of circular curve, length and shape of transition curve, longitudinal gradient, and radius of vertical curve. The line can be defined as three-dimensional longitudinal axis of track with characteristic changing of coordinates in space.

The first and the most important parameter of the line is radius of circular curve $R$. The main characteristic of horizontal circular curve is that its radius and cant have the constant values. The basic tendency in the track design is to avoid curves with small radius. It causes many harmful effects on wheel-rail contact forces, running safety, wear, energy consumption, cost-effectiveness, etc. The second parameter of the line is length and shape of transition curve which may have linear or parabolic change of cant from zero to the value of cant in circular curve. The radius of the transition curve is changing continuously between the tangent track and circular curve. The changes of cant and radius in transition curve have the same start and end position. The third parameter of the line is longitudinal gradient which is limited due to the characteristics of traction and braking. During the running on the uphill, there are increased wear of traction vehicles and energy consumption. On the other hand, during the running on the downhill, there are intensive wear of braking elements and thermal problems. The last, fourth parameter of the line is radius of vertical curve which connects the ends of the track in transition from one longitudinal gradient to another. While vehicles passing across the connections there are intensive change of accelerations and wheel-rail contact forces, which is very unfavourable for ride comfort and running stability. This parameter is limited in according to the maximal operating speed on given railway line and maximal vertical acceleration during the passing across connections.

\subsection{Real track geometry}

It is very important to note that the term "track geometry" always means the actual geometry or real track geometry in the exploitation. For proper evaluation of the track condition and analysis of dynamic behaviour of railway vehicles is very important to knowing the real track geometry. It is determined by measurement of deviations from the nominal geometry using specially equipped vehicles which is defined by the international standards EN13848. The parameters (deviations) which are measured are given in Table 1.

Table 1. The deviations from the nominal track geometry

\begin{tabular}{|l|l|}
\hline \multicolumn{1}{|c|}{ Parameter } & \multicolumn{1}{c|}{ Remark } \\
\hline $\begin{array}{l}\text { Deviations in longitudinal } \\
\text { direction in vertical plane }\end{array}$ & $\begin{array}{l}\text { Mean value of vertical } \\
\text { deviations of left and right rails }\end{array}$ \\
\hline $\begin{array}{l}\text { Deviations in lateral } \\
\text { direction in horizontal plane }\end{array}$ & $\begin{array}{l}\text { Mean value of lateral deviations } \\
\text { of left and right rails }\end{array}$ \\
\hline Deviations of track gauge & $\begin{array}{l}\text { Deviations from nominal track } \\
\text { gauge }\end{array}$ \\
\hline Deviations of cant & $\begin{array}{l}\text { Deviations from nominal cant } \\
\text { of outer rail in curve }\end{array}$ \\
\hline Twist & $\begin{array}{l}\text { Difference of cant between two } \\
\text { cross section of track divided by } \\
\text { certain distance }\end{array}$ \\
\hline
\end{tabular}

The track condition must be monitored in order to ensure timely response in case of exceeding the allowable values of deviations from the nominal geometry. The most of Railways classify their tracks just in accordance with these deviations. According to the international standards UIC 518 and EN14363, in terms of criteria of maintenance and eligibility for testing of railway vehicles, there are three classes of track quality $[11,12]$ :

- QN1: Require monitoring of condition of track or taking maintenance within regularly planned maintenance operations,

- QN2: Require short term maintenance operations,

- QN3: Characteristic for sections whose quality deviates from usual, but it is still acceptable from the standpoint of maintenance. If it is exceeded, the observed section is excluded from analysis of dynamic behaviour of railway vehicles.

\subsection{Rail profile}

The rail profile has significant influence on the dynamic of railway vehicles. On the most European railway lines, UIC60 rail is used (Fig. 2).

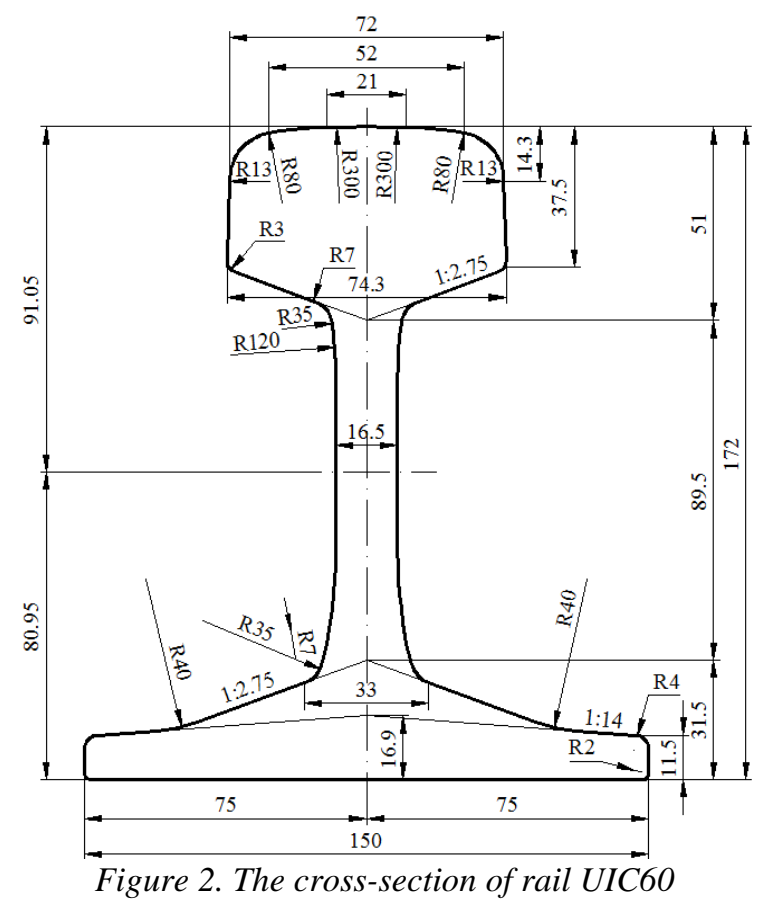

Depending on the permissible axle load and operating speed, the rails UIC 54, UIC 49, etc., are also used. During the exploitation, a very intense interaction with wheels leads to the wear of rail profile which can be uniform and non-uniform. The uniform wear implies that rail is worn over entire length, while non-uniform wear implies that rail has discrete damages at certain places. The most intensive uniform wear appears in curves where the highest values of wheel-rail contact forces is present. The typical worn profiles of outer and inner rail in curve is shown in Fig. 3.

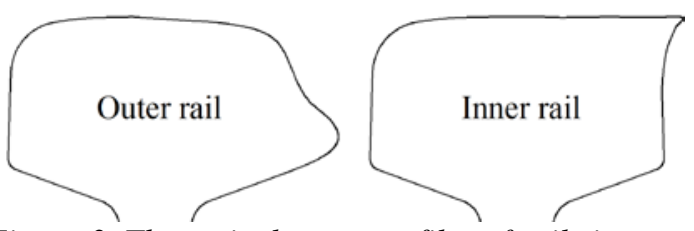

Figure 3. The typical worn profiles of rails in curve 
For evaluation of track condition and precise dynamic analysis is very important to determine the real rail profile. This is usually done using the special measuring devices - profile-meters. It is important to note that wear of the rail head leads to the increasing of track gauge. Non-uniform wear is caused by different factors. It is characterized by small damages or corrugations of the rail head surface which cause very unfavourable oscillations and noise during the passing of vehicles.

\section{PARAMETERS OF WHEELSET GEOMETRY}

As in the case of the track, it is very important to make difference between the nominal and real wheelset geometry (geometry of wheelset in exploitation). The international standards define nominal wheelset geometry and allowable tolerances which must be respected from all manufacturers. After a certain period of exploitation, intensive wheel-rail contact forces cause deviation from the nominal geometry. The parameters of the typical wheelset geometry are (Fig. 4):

- wheel radius (nominal rolling radius) $r_{0}$ (at normal track gauge $r_{0}=300 \div 600 \mathrm{~mm}$ ),

- distance between nominal rolling radii $2 b_{0}$ (at normal track gauge $2 b_{0}=1500 \mathrm{~mm}$ ),

- inner distance between wheels $2 b_{a}$ (at normal track gauge $2 b_{a}=1360 \mathrm{~mm}$ ),

- outer distance between flanges of wheels $2 b_{f}$,

- distance between axle-boxes $2 b_{l}$ (at normal track gauge $\left.2 b_{l}=2000 \mathrm{~mm}\right)$,

- flange height $h_{f}$ (at unworn wheels $h_{f}=28 \mathrm{~mm}$ ),

- flange thickness $t_{f}$ (at unworn wheels $t_{f}=32,5 \mathrm{~mm}$ ).

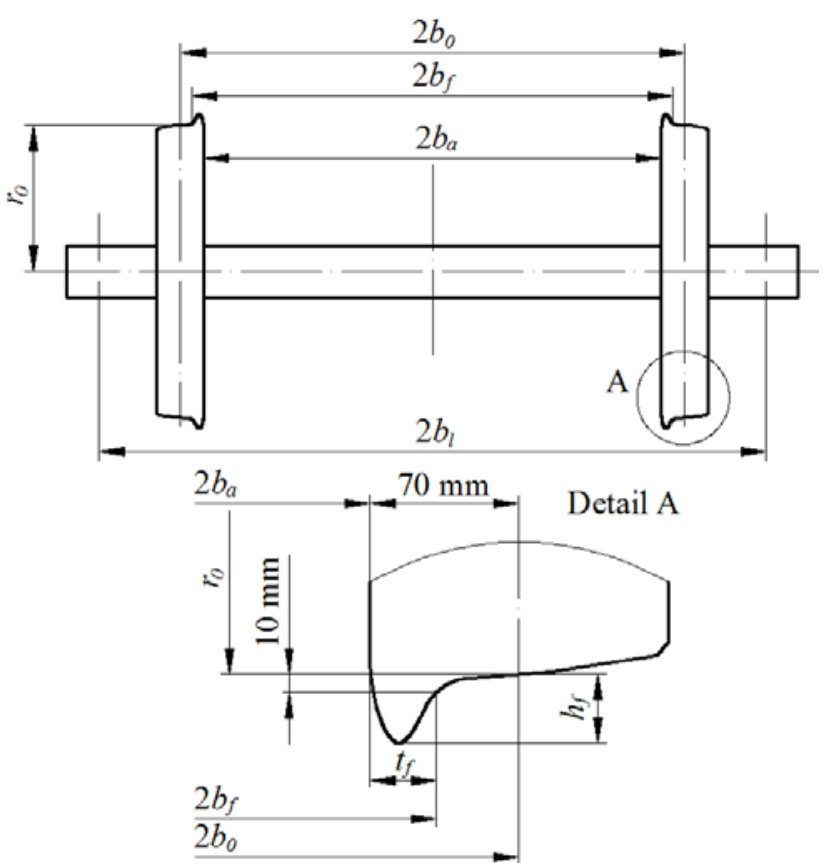

Figure 4. The parameters of wheelset geometry

During the running, the wheelset is exposed to a very intensive static, dynamic and thermal loads due to the interaction with the rail, braking, etc. This cause the wear whereby some of the mentioned parameters are changed. The most intensive wear is present on the wheel tread and flange, while the nominal wheel radius is decreased. The wheelset condition must be periodically observed during the exploitation with the aim to detect defects or exceeding the allowable values of deviations from the nominal geometry. Allowable values of wheelset and wheel wear are defined by the standard UIC510-2 [13].

\subsection{Wheel profile}

The wheel profile has a large influence on the ride comfort and running safety of railway vehicles. All wheel profiles have conical tread which provides steering of wheelset and passing through curves. One of the most used is profile UIC-ERRI S1002 which is shown in Fig. 5 [13].

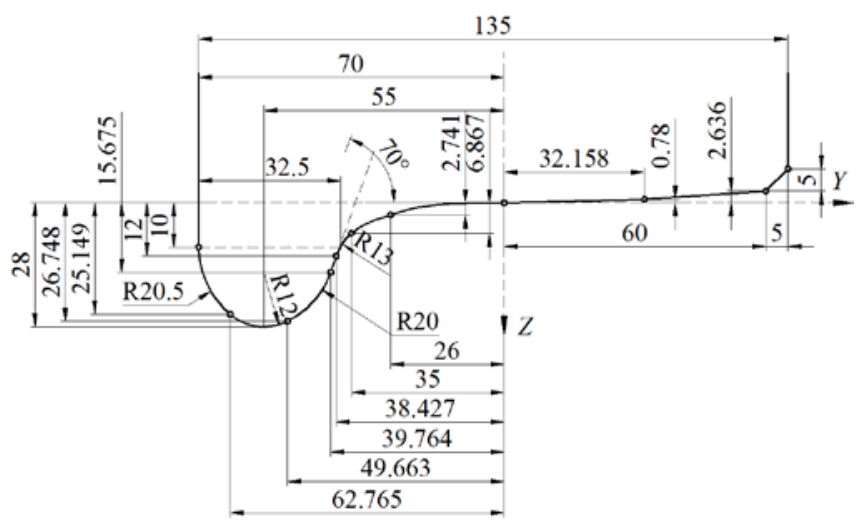

Figure 5. The nominal wheel profile UIC-ERRI S1002

It is very important to make difference between the new (unworn) and worn wheel profile. If the new wheel has a linear profile with straight cone, after certain period of exploitation it will be changed into the non-linear worn profile (uniform wear shown in Fig. 6.). After $100 \div 200$ thousands of mileage each worn profile has typical shape independently from the type of starting new wheel profile [2]. Thus, in order to reduce wear and its influence on the dynamic behaviour of railway vehicles, new wheels have profiles that are adopted to wear (Fig. 5).

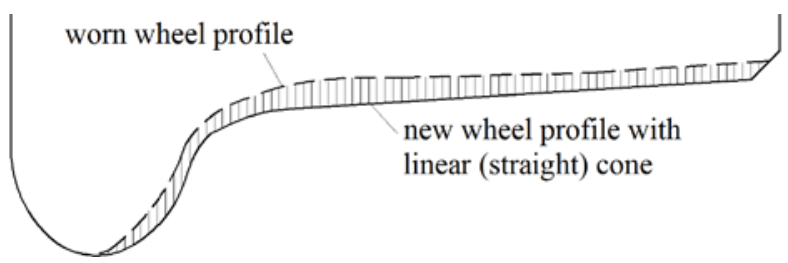

Figure 6. The uniform wear of wheel profile

In addition to the uniform, there is non-uniform wear which is consequence of different influences which cause the discrete damages (flats, dents, corrugations, peeling of material on the tread, damages of flange, eccentricity of wheel, etc.). For precise dynamic analysis and evaluation of wheel condition is very important to determine the real wheel profile by special profile-meters.

\section{PARAMETERS OF WHEEL-RAIL CONTACT GEOMETRY}

The parameters of wheel-rail contact geometry are shape of the wheel profile, shape of the rail profile, and geometrical sizes which define their relative position (Fig. 7). Geometrical sizes that define relative position of wheel profile in relation to the rail profile are: track gauge $G$; rail inclination; inner distance between wheels $2 b_{a}$; outer distance between flanges of wheels $2 b_{f}$; and lateral clearance of wheelset on track $\Delta b$.

\subsection{Track gauge}

The track gauge has a large influence on the relative positions between wheel and rail profiles. Due to 
the wear, track gauge increases and in that way changing the boundaries of the possible lateral displacement of the wheelset on the track. For more precise analysis of railway vehicles dynamic is very important to measure the real track gauge in function of longitudinal track position.

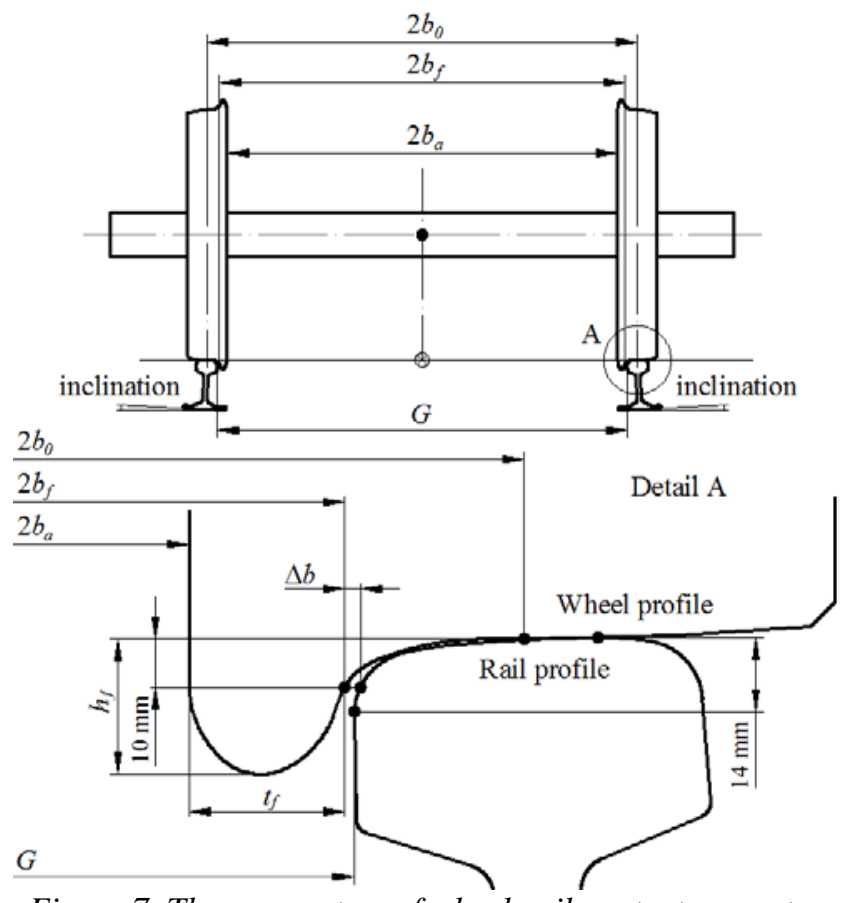

Figure 7. The parameters of wheel-rail contact geometry

\subsection{Rail inclination}

The rail inclination has an influence on the relative position between wheel and rail profiles only in the case of track with new rails which are placed exactly at certain transverse gradient $(1: 20,1: 40$, etc.) relative to the reference horizontal plane, and which have new unworn profiles. If rails are in exploitation for a longer time, their nominal (initial) profile is degraded, so inclination has no influence on the relative position between wheel and rail profiles. For precise analysis is necessary to measure worn rail profile in relation to the some reference coordinate system. In this way the real inclination is taken into account via the rail profile.

\subsection{Inner distance between wheels}

The inner distance between wheels directly affects the relative position of the wheel profile in relation to the rail profile. During the exploitation this parameter is less susceptible to changes due to the wear, but large deviations are possible due to the presence of eccentricity or similar damages of the wheels.

\subsection{Outer distance between flanges of wheels}

This parameter can be calculated based on the values of inner distance between wheels $2 b_{a}$ and flange thickness of left and right wheel $t_{f l}$ and $t_{f r}$ as follows:

$$
2 b_{f}=2 b_{a}+t_{f l}+t_{f r}
$$

Specially, if the flange thicknesses on the left and right wheel are identical then:

$$
2 b_{f}=2 b_{a}+2 t_{f}
$$

Due to the wear, the flanges thickness are decreased, so outer distance between flanges of wheels is also decreased. For a more precise dynamic analysis is very important to determine the real value of this parameter.

\subsection{Lateral clearance of wheelset on track}

When the wheelset is located in a central position on the track, between the wheel flange and rail head there is lateral clearance $\Delta b$ which is measured $10 \mathrm{~mm}$ below the nominal rolling radius (Fig. 7). Within this clearance, wheelset can be moved from central position to the left and right side, until the wheel flanges come into contact with the rail heads. The value of $\Delta b$ depends on the track gauge $G$ and the outer distance between flanges of wheels $2 b_{f}$. Due to the wear, the lateral clearance is increased, so for exact dynamic analysis is very important to determine the real values of this parameter.

\section{TYPES OF WHEEL-RAIL CONTACT}

During the running, the constant change of parameters of geometry which define the relative position of wheel profile in relation to the rail profile causes that location of contact point between them is constantly changing. In reality, wheel-rail contact is realized over a certain area which usually has the shape of ellipse. So, in further considerations when is used the term "contact point", it refers to the contact area and point in its centre. The contact point position at any time during the running depends on the shapes of wheel and rail profiles, as well as their relative position. The two typical cases of wheel-rail contact are tread contact and flange contact (Fig. 8).

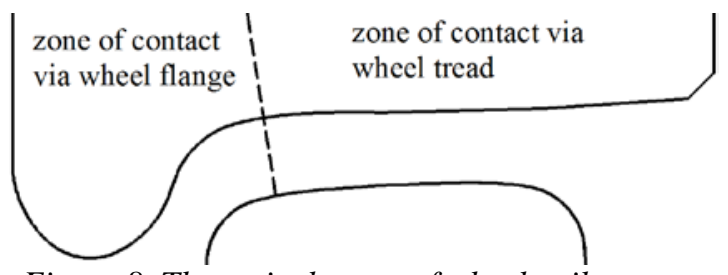

Figure 8. The typical zones of wheel-rail contact

\subsection{Tread contact}

The contact via the wheel tread is characteristic for running on tangent track or curves with higher radii. In this case, there is always one contact point, independently of the shapes of the wheel and rail profiles. If wheel and rail have worn profiles or profiles adopted to wear, contact point is continuously moving along the wheel and rail profiles, depending on the relative lateral displacement of wheel in relation to the rail. If the wheel and rail have other profiles (e.g. wheel profile with straight cone, etc.), contact point does not have to move continuously along the wheel and rail profiles, but can suddenly jump from one place to another. For wheel profiles UIC-ERRI S1002 and straight cone, in combination with the rail profile adopted to wear UIC 60, the cases of tread contact are shown in Fig. 9.

It is very important to note that contact via the wheel tread belongs to the so-called non-conformal contacts. At these contacts, radii of curvature of wheel and rail profiles in contact points are more or less different, which cause that in very small contact areas there are very intensive loads. This is especially typical for the new profiles of the rails and wheels with straight cone. 


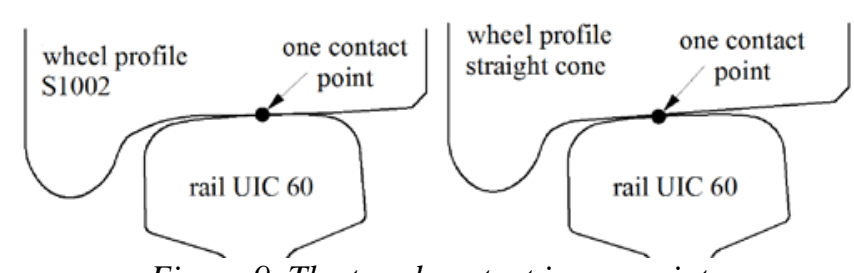

Figure 9. The tread contact in one point

\subsection{Flange contact}

Contact via the wheel flange is typical for running in sharp curves or in specific cases where there are larger deviations from the nominal track geometry. At contact via the wheel flange the following situations are possible: one contact point, two contact point, and conformal contact. If the wheel and rail have worn profiles or profiles adopted to wear, flange contact is realized via the one contact point, which is continuously moving along the wheel and rail profiles. On the other hand, if the wheel and rail have some other profiles (e.g. wheel profile with straight cone, etc.), flange contact can be realized via the one or two contact points, which can suddenly jump from one place to another. For wheel profiles S1002 and straight cone, in combination with the rail profile adopted to wear UIC60, the cases of flange contact are shown in Fig. 10.

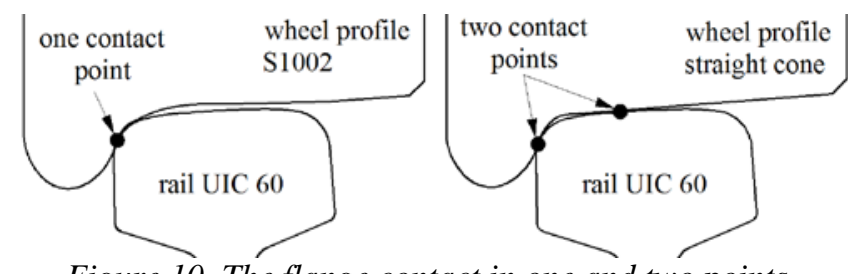

Figure 10. The flange contact in one and two points

The conformal contact exist when surfaces over which the wheel and rail are in touch are equal or very similar, or when the radii of curvature of wheel and rail profiles in contact point are equal or very similar. In such case the contact is realized via a larger contact area which is greater if the surfaces in touch are more conformal. The conformal contact is typical for transition between tread and flange, and worn wheel and rail profiles (Fig. 11).

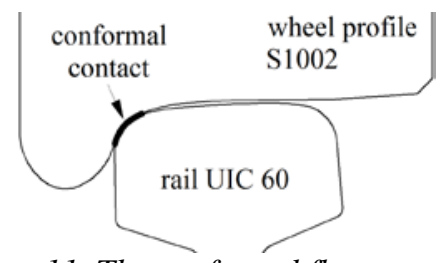

Figure 11. The conformal flange contact

\section{FUNCTIONS OF WHEEL-RAIL CONTACT GEOMETRY}

During the running, wheelset is moving in lateral direction in relation to the track centre line. The maximal lateral displacement $\Delta y$ are limited by the wheel flanges contacts with rail heads. At a certain lateral displacement $\Delta y$, the mutual positions of wheels and rails profiles are changed, as well as the following geometric sizes in contact of left and right wheels and rails (Fig. 12): contact points positions; rolling radii in contact points on left and right wheel $r_{l}$ and $r_{d}$; contact angles in contact points on left and right wheel $\gamma_{l}$ and $\gamma_{d}$; and rotation angle of the wheelset around longitudinal axis of track (roll angle) $\varphi$.

In addition, the radii of touching surfaces are changed, which cause the changing the shape and size of contact areas. The changes of all mentioned geometric sizes are depend on the parameters of wheel-rail contact geometry and are expressed as a function of wheelset lateral displacement in relation to the track centre line $\Delta y$.

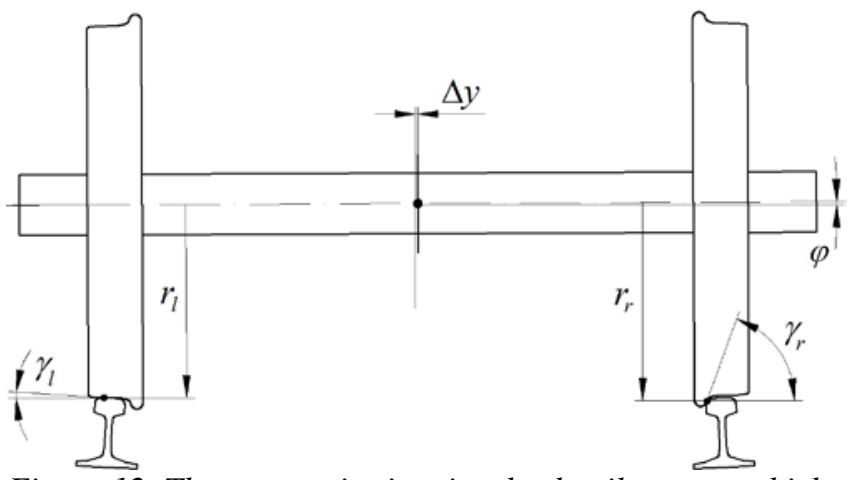

Figure 12. The geometric sizes in wheel-rail contact which are changed at lateral displacement of wheelset

Since in practice there are various combinations of profiles of wheels and rails, in order to easier characterization and analysis, the functions of wheel-rail contact geometry are introduced. Suppose that, in some point of time during the running, the wheel is moved laterally in relation to the rail for value $\Delta y$. At the same time, there is a raising of wheel for value $\Delta z$, increasing of rolling radius for value $\Delta r$, and changing of contact angle $\gamma$, as shown in Fig. 13. The changes of these three sizes in function of wheel lateral displacement are called wheelrail contact geometry functions or contact functions [2]. The contact functions have strongly nonlinear character and are defined as:

$$
\begin{aligned}
& \Delta r=f(\Delta y) \\
& \Delta z=g(\Delta y) \\
& \gamma=h(\Delta y)
\end{aligned}
$$

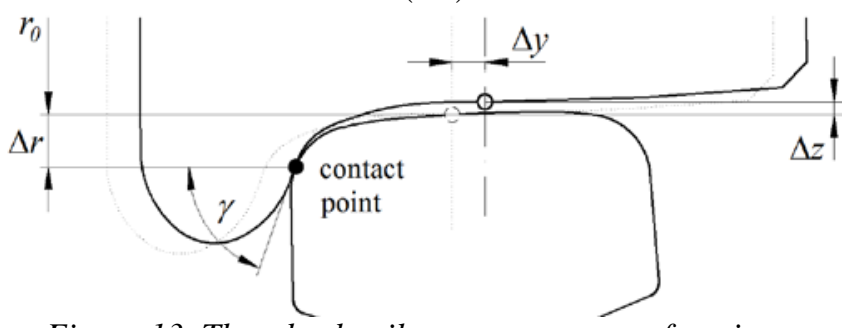

Figure 13. The wheel-rail contact geometry functions

In the expressions (4-6) $f, g$, and $h$ are functions that depend on the parameters of wheel-rail contact geometry, especially the shapes of wheel and rail profiles. If contact point is continuously moving over the wheel profile, the contact functions are continuous. If contact point suddenly jumping from one place to another over the wheel profile, the contact functions have interruptions.

For practical characterization of wheel-rail contact geometry another function is used. It's about equivalent conicity which is determined for different combinations of wheel and rail profiles and different parameters of wheelrail contact geometry.

\subsection{Conicity}

At the beginning of development of railways, the wheels had profiles with straight cone. The profile was linear, usually with cone $1: 20$, which was in correlation with the rail inclination, which was also 1:20. For 
modelling of such wheel-rail contact geometry, the simplified model shown in Fig. 14 is usually analysed. It is assumed that wheels have identical conical profiles which are rolling on the rails whose profiles are circular. Also it is assumed that there is no contact between the wheel flanges and rail heads. In this case, there is only one contact point between wheel and rail profiles.

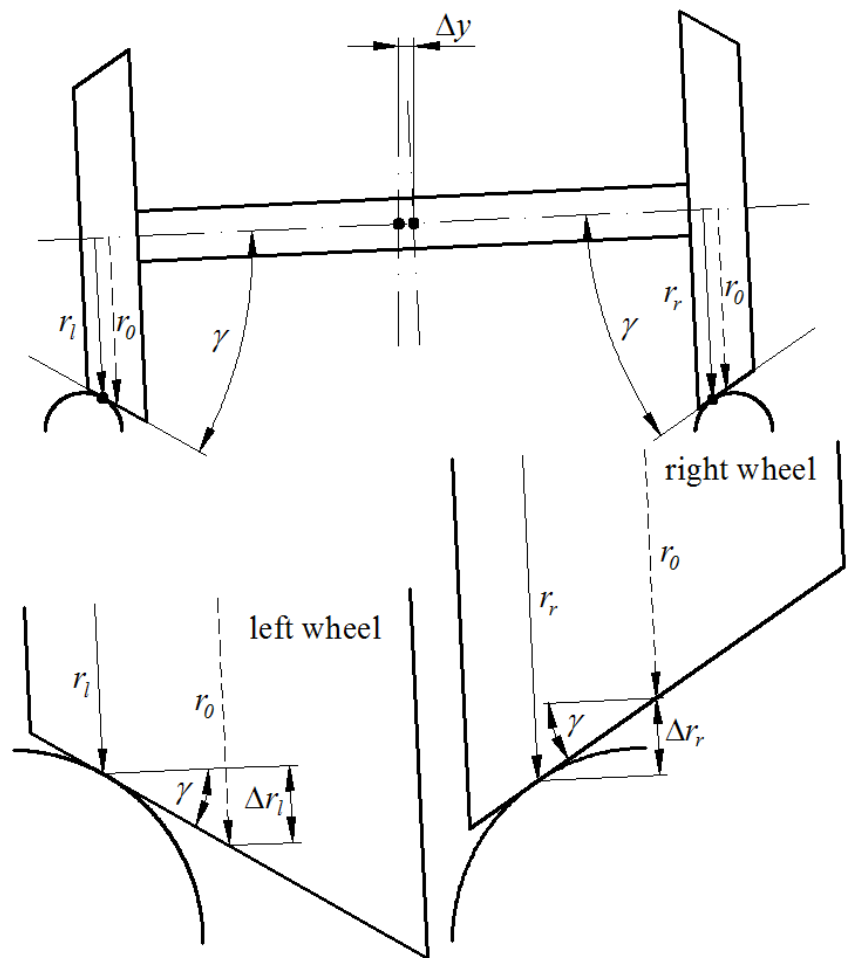

Figure 14. The wheel-rail contact geometry - wheel profile with straight cone and circular rail profile

When the wheelset is located in central position on the track, rolling radii of the left and right wheel are equal:

$$
r_{l}=r_{r}=r
$$

If the wheelset is moved laterally for value $\Delta y$, the changes of rolling radii on the left and right wheel will be $\Delta r_{l}$ and $\Delta r_{r}$, respectively. The total difference between rolling radii on the left and right wheel is:

$$
\Delta r=\Delta r_{l}+\Delta r_{r}
$$

Since the profiles of the wheels have linear shape and equal conicity, these two changes are equal:

$$
\Delta r_{l}=\Delta r_{r}=\Delta r / 2
$$

The rolling radii of left and right wheel are:

$$
\begin{aligned}
& r_{l}=r_{0}-\Delta r_{l} \\
& r_{r}=r_{0}+\Delta r_{r}
\end{aligned}
$$

Substituting the expression (9) in the previous expressions is obtained:

$$
\begin{aligned}
& r_{l}=r_{0}-\Delta r / 2 \\
& r_{r}=r_{0}+\Delta r / 2
\end{aligned}
$$

Therefore, the difference between the rolling radii of the left and the right wheel is:

$$
\Delta r=r_{r}-r_{l}
$$

Since at any given time the difference between the rolling radii depends on the lateral displacement of wheelset centre, it can be written:

$$
\Delta r(y)=r_{r}(y)-r_{l}(y)
$$

From Fig. 16 the following relation can be seen:

$$
\tan \gamma=\frac{\Delta r_{l}}{\Delta y}=\frac{\Delta r_{r}}{\Delta y}
$$

Substituting the expression (9) in the previous expression (16) is obtained:

$$
\tan \gamma=\frac{\Delta r}{2} \frac{1}{\Delta y}
$$

Based on that, function $\Delta r$ can be expressed as:

$$
\Delta r=2 \cdot \Delta y \cdot \tan \gamma
$$

For small angles $\gamma$ (as is the case with the wheel profile with straight cone $1: 20$ ) is $\tan \gamma \approx \gamma$, so the following relation is obtained:

$$
\Delta r=2 \cdot \Delta y \cdot \gamma(y)
$$

In this way, the relation between lateral displacement of the wheelset $\Delta y$, conicity $\gamma$, and rolling radii difference $\Delta r$, is established through function (19). This function is, for wheelset with wheels profiles with straight cone, a linear function which describes wheel-rail contact geometry for any lateral position of wheelset on the track. The change of function (19) for bi-linear wheel profiles (two straight cones with different slopes, one in tread and one in flange zone) is shown in Fig. 15.

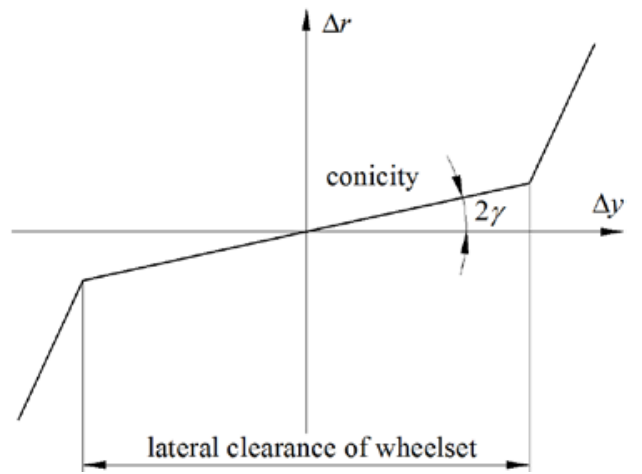

Figure 15. The change of rolling radius in function of wheelset lateral movement (wheels profiles with straight cone)

\subsection{Equivalent conicity}

In contrast to the wheels profiles with straight cone, in practice there are worn wheel profiles or profiles adopted to wear. These profiles are non-linear, so the previously exposed methodology based on constant conicity is not applicable for analysis and characterization of such wheel-rail contact geometry. In this case, the difference between rolling radii will not be the same as in the case of wheels with straight cone (Fig. 16).

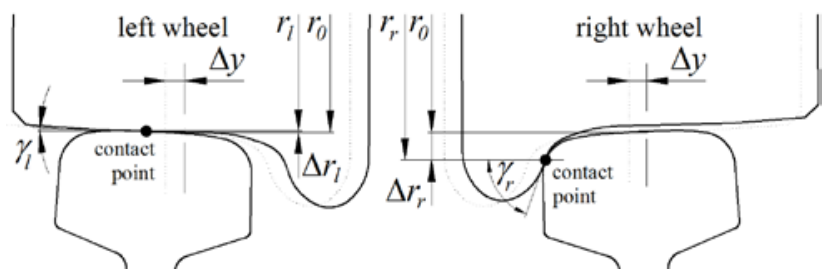

Figure 16. The wheel-rail contact geometry - worn wheel profile

For the case in Fig. 16, the difference between the rolling radii of the left and the right wheel is:

$$
\Delta r=r_{r}-r_{l}
$$

Wherein:

$$
r_{r}=r_{0}+\Delta r_{r}
$$




$$
r_{l}=r_{0}-\Delta r_{l}
$$

The difference between the rolling radii depends on the lateral displacement of wheelset, and based on that is:

$$
\Delta r(y)=r_{r}(y)-r_{l}(y)
$$

However, this function does not have a linear character, but is strictly a nonlinear function. In that sense, the key question is how to describe the geometry of the wheel-rail contact for non-linear worn wheel profiles. For this reason, and based on previous analysis of the conicity of wheels with straight cones expressed via the equation (19), the so-called equivalent conicity is introduced:

$$
\lambda_{e}=\frac{\Delta r}{2 \cdot \Delta y}
$$

Substituting the expression (20) in the previous expression is obtained:

$$
\lambda_{e}=\frac{r_{r}-r_{l}}{2 \cdot \Delta y}
$$

At the end, substituting the expression (21) and (22) in the previous expression (25) is obtained:

$$
\lambda_{e}=\frac{\Delta r_{r}-\Delta r_{l}}{2 \cdot \Delta y}
$$

The equivalent conicity $\lambda_{e}$ for wheels with worn profiles has the same physical meaning as conicity $\gamma$ for wheels with straight cones. Practically, it is linearization of nonlinear changes of rolling radii of wheels at a certain wheelset lateral displacement $\Delta y= \pm y^{\prime}$ (Fig. 17).

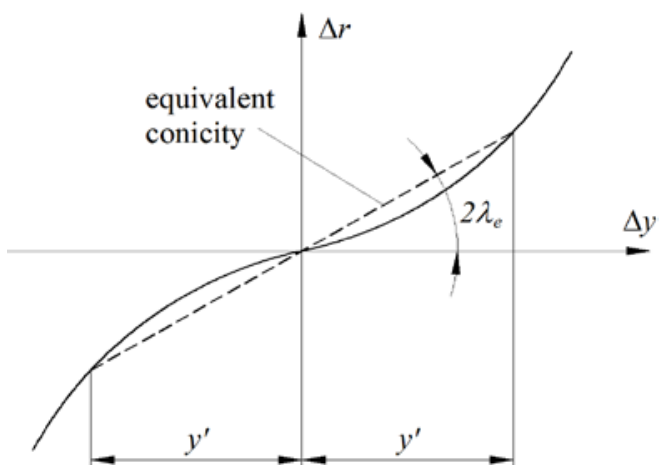

Figure 17. The change of rolling radius in function of wheelset lateral movement (worn wheel profile)

Equivalent conicity for the wheel profiles with straight cone is equal to the conicity $\lambda_{e}=\gamma$. It is very important to emphasize that due to the wear, all linear profiles with straight cone after a certain period of exploitation becomes worn and nonlinear. On the other hand, optimization from the aspects of wear and improving the dynamic behaviour of railway vehicles is leads to the optimal wheel profiles or profiles adopted to wear. Such profiles with nonlinear shape (Fig. 5) are today most used in production of new wheels and wheelsets. Based on this it can be concluded that for describing and analysing the wheel-rail contact geometry of most wheelsets and tracks in exploitation is necessary to use the equivalent conicity. The main parameters influencing the equivalent conicity are: wheel profile; rail profile; relative lateral displacement of wheelset $\Delta y$; track gauge $G$; inner distance between wheels $2 b_{a}$; flange thickness $t_{f}$; and rail inclination.

The considered constant change and difference in rolling radii on the left and right wheel causes the sinusoidal motion of the wheelset (Fig. 18). The oscillations caused by sinusoidal motion are the most unfavourable effects in running of railway vehicles on the tangent track and curve with large radii. Besides very negative influence on ride comfort, they can cause vehicle instability at certain critical speeds and leads to the derailment with huge consequence. That is why the sinusoidal motion is key influential parameter on the lateral dynamic stability and its analysis is one of the most important task in rail vehicle dynamics.

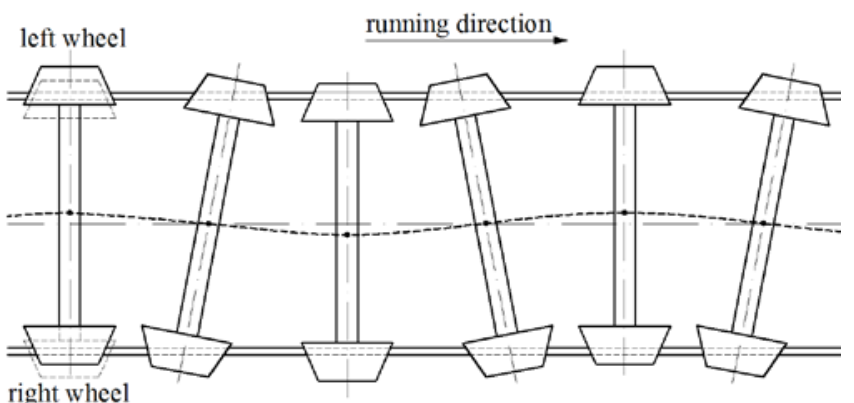

Figure 18. The sinusoidal motion of wheelset

The sinusoidal motion is described by Klingel equation which establishes relation between the wavelength of sinusoidal oscillation of wheelset centre $L_{w}$, half distance between nominal rolling radii $b_{0}$, nominal rolling radius $r_{0}$, and equivalent conicity $\lambda_{e}$ (or conicity $\gamma$ for wheels profiles with straight cones) [14]:

$$
L_{w}=2 \pi \sqrt{\frac{b_{0} \cdot r_{0}}{\lambda_{e}}}
$$

As can be seen from Klingel's equation, the equivalent conicity is one of the dominant influential parameters on the frequency of sinusoidal oscillations. In that sense, the standard UIC 518 defines the limit values of equivalent conicity from the point of maximal operating speed and running safety [11].

Bearing in mind the non-linearity and stochasticity of a large number of influential parameters, accurately determining the equivalent conicity is a very complex task. Depending on the given wheelset lateral displacement, for determination of equivalent conicity three methods can be used [2].

The first and the most simplest method involves the determination of equivalent conicity using equations (2628), whereby the difference between the rolling radii at only one amplitude of the lateral displacement is taken into account. This is usually the maximal amplitude $\Delta y= \pm 4$ $\mathrm{mm}$. The method does not include the non-linearity in the range of changes in the amplitude of lateral displacement.

The second method involves the determination of equivalent conicity using equations (26-28), whereby a few amplitudes of lateral displacement are taken into account. For example, these amplitudes can be $\Delta y= \pm 1, \pm 2$, \pm 3 , and $\pm 4 \mathrm{~mm}$. It provides more accurate results and a much better picture about changes of equivalent conicity.

Third, the most complicated way involves the determination of equivalent conicity according to the method proposed in the standards UIC 519 and EN15302 $[15,16]$. According to this method, the wavelength of oscillations of wheelset sinusoidal motion on given track is determined or measured, at amplitude of lateral displacement $\Delta y= \pm 3 \mathrm{~mm}$. After the determination of 
wavelength, the equivalent conicity is determined by Klingel equation (27). This practically means that the equivalent conicity of nonlinear worn profile is defined as a certain conicity of linear profile which, at a certain lateral displacement of wheelset, gives the equal wavelength of sinusoidal oscillations as nonlinear worn profile. In this way, the nonlinear change of rolling radii in range of changes of amplitude of lateral displacement is taken into account. It is very important to emphasize that all three methods provide a single value of equivalent conicity for given or assumed value of wheelset lateral displacement in relation to the central position on the track. These methods are only attempts to describe a very complex geometrical relationships that exist in the wheelrail contact. For a more accurate dynamic analysis is very important to analyse the influence of nonlinear geometrical relationships in the wheel-rail contact on the dynamic behaviour of railway vehicles. Consequently, it may be of large interest to improve the way of current description and characterization of wheel-rail contact geometry.

\section{CONCLUSION}

This paper gives a systematic approach to the analysis of wheelset-track and wheel-rail contact geometry. In first phase, the parameters of wheelset and track geometry are introduced, while particular attention is paid to the geometry of wheel and rail profiles. In the second phase, the interaction wheelset-track and wheel-rail is analysed. The special attention is paid to the nonlinear geometric relationships between wheel and rail profiles. This led to the defining the parameters of wheel-rail contact geometry. In the final, third stage, the functions which combines all analysed parameters are derived. The emphasis is placed on the deriving the expressions for conicity and equivalent conicity. The methods of determination of equivalent conicity and its influence on the dynamic behaviour of wheelset and railway vehicles are discussed. Its correlation with the most important phenomenon of running of railway vehicles on the tangent track - sinusoidal motion of wheelsets, is established and analysed. Considerations in the paper confirm the fact that wheel-rail contact geometry and equivalent conicty as function which representing its characterization, belong to the most important influential parameters on the dynamic behavior of railway vehicles.

\section{ACKNOWLEDGEMENTS}

The authors express their gratitude to Serbian Ministry of Education, Science and Technological Development for supporting this paper through project TR35038.

\section{REFERENCES}

[1] S.D. Iwnicki, "Handbook of Railway Vehicle Dynamics”, CRC Press, Taylor \& Francis Group, Boca Raton (USA), (2006)

[2] E. Andersson, M. Berg, S. Stichel, "Rail Vehicle Dynamics”, Railway Group KTH, Stockholm (Sweden), (2007)
[3] I.Y. Shevtsov, "Wheel/Rail Interface Optimisation", TU Delft, Delft (Holland), (2008)

[4] J. Gerlici, T. Lack, "Contact geometry influence on the rail/wheel surface stress distribution”, Procedia Engineering, 2, p. 2249-2257, (2010)

[5] S. Mace, R. Pena, N. Wilson, D. DiBrito, "Effects of wheel-rail contact geometry on wheel set steering forces”, Wear, 191, p. 204-209, (1996)

[6] O. Polach, "Influence of wheel/rail contact geometry on the behaviour of a railway vehicle at stability limit”, Proceedings of 5th EUROMECH Nonlinear Oscillations Conference - ENOC-5, Eindhoven (Netherlands), 7 August-12 August, p. 2203-2210, (2005)

[7] D. Baldovin, S. Baldovin, "The Influence of the Wheel Conicity and the Creep Force Coefficients to the Hunting Motion Stability of a Bogie with Independently Rotating Wheelsets”, Revue Roumaine des Sciences Techniques - Série de Mécanique Appliquée, 56, No. 1, p. 11-19, (2011)

[8] J. Sadeghi, B. Akbari, "Field investigation on effects of railway track geometric parameters on rail wear", Journal of Zhejiang University SCIENCE A, 7(11), p. 1846-1855, (2006)

[9] R. Jandora, "Modelling of railway vehicle movement considering non-ideal geometry of wheels and rails", Applied and Computational Mechanics, 1, p. 489498, (2007)

[10] O. Polach, "Characteristic parameters of nonlinear wheel/rail contact geometry”, Vehicle System Dynamics: International Journal of Vehicle Mechanics and Mobility, Vol. 48, Suppl. 1, Special Issue: IAVSD Supplement, p. 19-36, (2010)

[11] UIC CODE 518 OR, “Testing and approval of Railway vehicles from the point of view of their dynamic behavior - Safety - Track fatigue Running behavior”, 4th edition, International Union of Railways - UIC, (2009)

[12] EN 14363, "Railway applications - Testing for the acceptance of running characteristics of railway vehicles - Testing of running behavior and stationary tests”, European Committee for Standardization CEN, (2005)

[13] UIC CODE 510-2 OR, “Trailing stock: wheels and wheelsets. Condition concerning the use of wheels of various diameters", 4th edition, International Union of Railways - UIC, (2004)

[14] J. Klingel, “Uber den Lauf von Eisenbahnwagen auf gerader Bahn, Organ fur die Fortschritte des Eisenbahnwesens”, Neue Folge 20, 113-123, Tafel XXI, (1883)

[15] UIC CODE 519 OR, "Method for determining the equivalent conicity”, 1st edition, International Union of Railways - UIC, (2004)

[16] EN15302: "Railway applications - Method for determining the equivalent conicity", European Committee for Standardization - CEN, (2011) 